\title{
Two-Dimensional Construction Business Translation Teaching Mode for Females Based on Social Constructivism
}

\author{
Tan Shuxin ${ }^{1, \text { a }}$ \\ ${ }^{1}$ Department of TESOL, Graduate School of Education, Pai Chai University, Daejeon, Republic of Korea/ Department of \\ Literature, Hunan Women's University, Changsha, Hunan, China,410004 \\ a278835001@qq.com
}

\begin{abstract}
Guided by the theory of Social Constructivism, this paper constructs a two-dimensional Construction translation teaching mode consisted of cooperative construction and practical construction, which conforms to the characteristics of female gender and language learning style, it is concluded that this teaching model aims to help female students to learn business English translation and effectively improve their business English translation.
\end{abstract}

Keywords: Social Constructivism; Women's University; Business English Translation; Teaching mode

\section{基于社会建构主义的女性二维商务翻译教学模式}

\section{谭舒心 ${ }^{1, a}$}

${ }^{1}$ 湖南女子学院文学院, 长沙, 湖南, 中国, 410004

培材大学教育研究生院 TESOL 系, 大田, 韩国

a278835001@qq.com

\section{摘要}

本文以社会建构主义理论为指导，构建了一种由合作建构和实践建构共同构成的二维建构翻译教学模 式根据女性性别和语言学习方式的特点, 得出该教学模式旨在帮助女学生有效地学习商务英语翻译, 提高他们的商务英语翻译水平。

关键词: 社会建构主义; 女子大学; 商务英语翻译; 教学模式

\section{1. 引言}

商务英语翻译是商务英语专业的核心课程之一, 然 而当下普通高等院校采用的传统商务英语翻译教学模 式中存在一些弊端, 比如教学环境单一化、理论与实践 脱节、考核评价片面化等。因此, 近年来国内外不少学 者在传统翻译教学模式的基础上, 不断探寻新的翻译教 学思想, 其中建构主义理论被认为是最具前景学习理 论。目前, 我国社会建构主义翻译教学研究开始从理论 分析走向针对不同学习群体进行实证研究, 但该教学模 式的研究仍鲜见于女子大学。女子大学是以女性独特的 思维方式和不同的生活角色为依据建立起来的单性高 等教育模式, 因此针对女子大学传统商务翻译教学模式 的改进要考虑到女性性别特点与语言学习风格, 以此更 好开发女性学生商务翻译的潜能。

\section{2. 社会建构式女性翻译教学模式理念}

\section{1 社会建构主义理论}

社会建构主义兴起于 20 世纪 80 年代, 是建构主义 的重要流派。这一学派认为个体是从自身经验出发对客 观事物进行主观理解和意义建构, 即知识来源于环境的 交互作用, 学生不是通过教师的传授来获得知识, 而是 通过自身的主动建构获得。因此在社会建构主义看来, 学习本质上是参与者群体共同建构社会共享认知的过 程。 


\section{2 模式理念}

从性别论的视角看, 女性学习风格特点区别于男 性, 具体可归为听觉型、场依存型以及外向型。这三个 特点原本应是女性学生学习语言的优势, 但在传统模商 务翻译课堂中, 很多女性学生往往不太自信, 不愿主动 表现, 依赖标准答案, 被动消极, 无法利用好自身的语 言学习优势。因此, 在以女性为教学对象的商务翻译教 学实践中, 要充分利用女性学习风格, 让学生通过主动 合作学习, 克服消极思想, 建构知识, 取长补短, 最终 成为在学习过程中能够自我控制、自我调节, 具有自 我分析和评价能力、善于学习的终身学习者。

\section{3. 双阶建构式翻译作坊教学模式}

\section{1 以翻译作坊为形式的合作式建构}

翻译作坊指一群从事翻译活动的人聚集一起, 就某 项具体翻译任务进行见深入讨论, 协商议定出该群体成 员均认同译文的活动, 十分契合女性学习风格特点。在 正式开课前, 学生自行分组建立翻译作坊, 并推选负责 人。上课时, 教师先花 15 分钟常规教学, 补充知识。 然后通过翻译作坊引导学生进行合作建构。该部分分为 三个阶段: 在译前准备阶段, 给每个作坊分派限时翻译 任务, 要求各坊成员共同探讨翻译风格, 统一术语表达 等问题。在译中阶段, 成员独立翻译, 形成初稿。初稿 完成后进行交叉审译, 形成定稿。在译后阶段, 小组逐 一展示讨论交流, 教师总结归纳。之后组内根据反馈形 成终稿, 并根据翻译反思出具报告。

\section{2 以翻译项目为驱动的实践式建构}

本部分是教学中后期开展的课外实践活动, 主要是 基于前中期翻译作坊模式形成合作性建构的基础上, 通 过真实情景中的翻译项目实战展开实践式构建。笔者组 建了翻译小组, 并与某外贸公司开展校企合作, 获得真 实翻译项目。学生因为有前期的翻译作坊工作经验, 在 实战中能较好地开展合作翻译, 教师只需通过网络, 就 项目管理、质量把控、文本编辑方面给指导。翻译过程 中, 学生可以把组内难以解决的问题或存有疑虑的问题 发送到工作群, 师生讨论交流共同解决。项目完成后, 教师和企业对其进行综合评价, 各作坊根据反馈情况修 改译文, 完成最终译本, 并根据此次翻译实践情况进行 反思和总结，形成实践报告。

在 “翻译作坊” 教学模式设计中, 教师是该教学模 式的核心，扮演着 “集设计者、诱导者、研究者和管理 者于一身的 “主导” 角色（朱波，2011:53）, 而学生 则是该教学模式的中心, 通过在学习的过程中, 相互沟 通讨论、取长补短, 合作完成翻译任务, 使之对所学知 识有更全年、更深层次的理解。
通过合作式建构和自主式建构两个阶段，师生之 间、学生之间, 构建出一个相互独立却又联系紧密的合 作学习模型。

\section{4. 实验方法与说明}

\section{1 研究问题}

本研究具体考察以下问题:基于社会建构主义的女 性二维商务翻译教学模式是否有利于促进女性学生商 务翻译能力的发展。

\section{2 试验目的和试验对象}

此次选取了湖南女子学院商务英语专业大三两个 班级（共计 96 位学生）作为对照实验的测评对象, A 班（48 人）设定为实验班，B 班 (48 人) 设定为对照 班。经过前置测试, 确保实验对象 ( $\mathrm{AB}$ 两班学生) 整体 英语水平大致相当。实验条件配置统一, 确保实验课程 的授课教师、教材选用、学习环境具有统一性, 基本杜 绝无关变量。

\section{3 内容与过程}

本实验以《商务笔译》课程为实验课程, 实验教材 为对外经贸大学出版社的 《Business English Translation》。教学目标设置相同。A 班 (实验班) 采 用 “翻译作坊” 教学模式, 而 B 班 (对照班) 采用传统 授课模式。整个实验时长共 16 周, 进行了四次水平测 试, 分别设置在第 1 周、第 6 周、第 11 周、第 16 周, 其中第 1 周为摸底测试。

\section{4 实验数据分析}

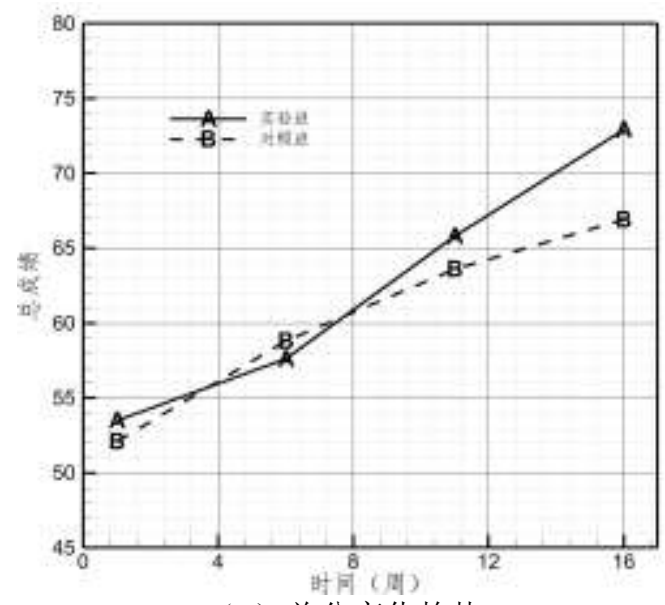

（a）总分变化趋势 


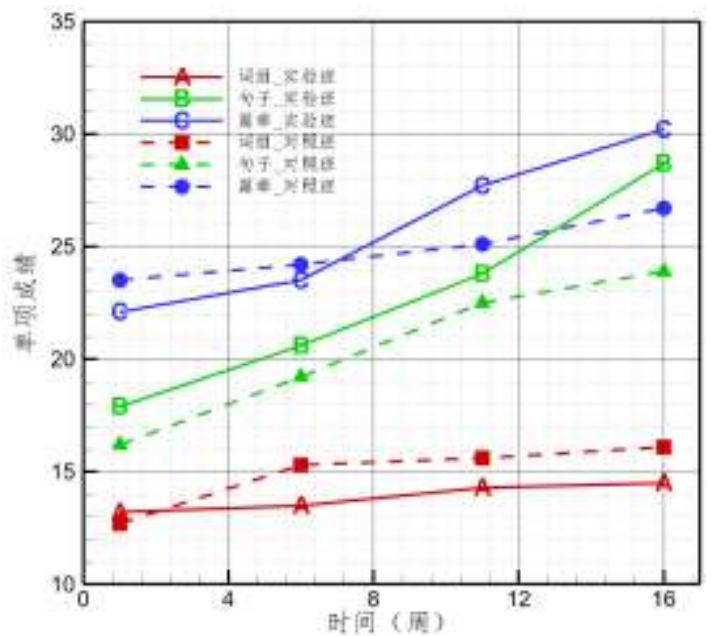

(b) 单项成绩变化趋势

图 1 成绩分析

统计两班 4 次测试成绩, 如图 1、图 2 所示, 其中 图 1 为总分随时间变化趋势对比, 图 2 为单项成绩随时 间变化趋势对比。

由图 1 可知, 摸底测试中 (第 1 周) 实验班成绩略 高于对照班, 两班成绩差异不大（显著性值 $P>0.05$ ）, 即两班在实验前的初始条件基本相同, 说明这两个班级 具有可比性。第二次测试时 (第 6 周) 实验班平均成绩 反而低于对照班, 结合学期结束后对学生的回访, 原因 是实验班同学在教学初期对新的教学模式持怀疑抵触 心理, 同时笔者对新的教学模式也缺少相关实践经验。 之后实验班同学逐渐适应并接受了新的教学模式, 师生 默契度提高, 在第三次测试时（第 11 周）, 实验班成 绩有了明显提高, 并超过了对照班成绩。经过一学期的 学习, 实验班在第四次 (第 16 周) 的测试中成绩大幅 领先对照班。由图 2 可知, 新的教学模式主要是通过提 高学生的句子翻译和篇章翻译能力从而提高整体翻译 水平, 而传统教学模式在词组翻译的水平提高上也有一 定的优势。

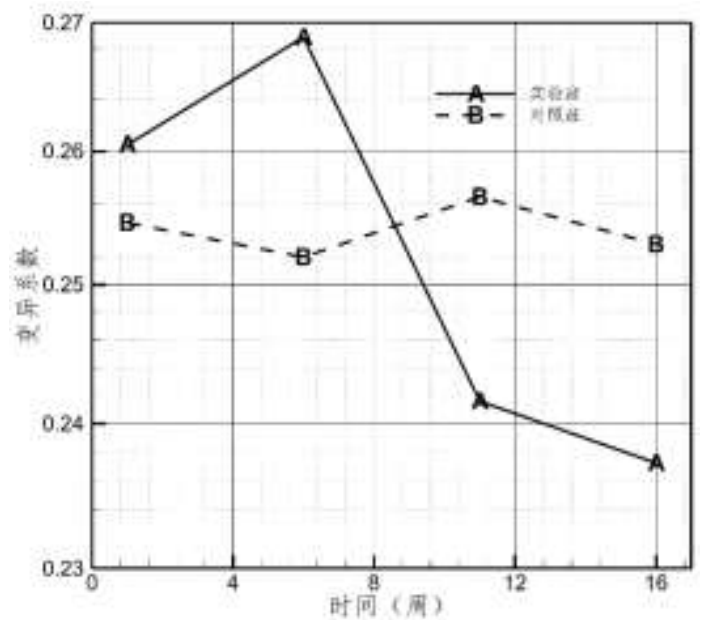

图 2 变异系数
平均成绩仅能反应整体水平，又因为每次考试平均 成绩不同, 单纯用标准差不能准确地比较数据的离散程 度, 为了解每个班级学生成绩的差异程度, 引入变异系 数 $\mathrm{CV}$ 。CV 是概率分布离散程度的归一化量度, 是衡量 数据中各观测值变异程度的一个统计量。

$C V=\frac{\sigma}{\bar{X}}=\sqrt{\frac{\sum_{i=1}^{n}\left(\mathrm{x}_{i}-x\right)^{2}}{n-1}} / \frac{\sum_{i=1}^{n} x_{i}}{n}$

图 3 变异系数 $\mathrm{CV}$ 计算公式

其中, $\sigma$ 是标准差, $\bar{X}$ 为平均差, $\mathrm{n}$ 为样本数量。 由图 2 可知, 传统教学模式提高学生成绩的同时, 学生之间的差异程度变化不大。而新的教学模式提高了 学生成绩的同时, 还降低了学生之间的差异程度。即传 统教学模式很难改变学生之间的既有差距, 而新的教学 模式通过合作学习, 缩小了学生之间的差距, 实现了共 同进步。值得注意的是，第二次测试时（第 6 周），实 验班的变异系数显著增大, 说明此时实验班同学对新的 教学模式的接受程度有较大差异, 有的很快适应了新的 教学模式并取得进步, 有的接受过程比较慢并没有取得 预想中的教学目标。因此, 在教学改革、推行新的教学 模式时要注意学生之间的个体差异, 因材施教, 才能达 到更好的教学效果。

\section{5. 结语}

本研究构建了一个契合女性学生性别特点及语言 学习风格的双阶建构式商务翻译教学模式。相比于传统 翻译教学模式, 新模式从合作性构建和实践性构建两个 维度来构建女性学生商务翻译综合能力, 引导学生转变 固有的学习理念, 克服自身惰性, 形成独立自主、具有 自我反思和批判性的翻译精神, 从而提高了其翻译实操 能力和团队合作能力。通过实验教学及数据分析, 初步 得出以下结论:

第一, 相较于传统教学模式, 新的教学模式有助于 女性学生商务翻译能力的建构, 提高商务翻译水平, 可 以缩减学生间差距, 实现共同进步;

第二, 新的教学模式下学生词组翻译水平不如传统 教学模式, 以后在教学实践中, 要注意吸收传统教学模 式的优点;

第三, 不同学生对新的教学模式接受能力不同, 要 根据个体差异，做到因材施教，量体裁衣。

\section{基金项目}

湖南女子学院校级课题（批准号, HNNY18YB KT008); 湖南省普通高校教学改革 项目研究（批准号, 湘教通 [2019]29-1136)); 
国家民委民族研究自筹项目（批准号, 2019-GMD-062）。

\section{REFERENCES}

[1] Kiraly, Don. A Social Constructivist Approach to Translator Education: Empowerment from theory to practice[J]. Target. 2004(1):157-162.

[2] Kaeryna Kozlova. Kolb's Learning Styles: Preferences among Male and Female Students of

English for Specific Purposes (ESP)[J]. Human and Social Studies. 2018 (1)37-39.
[3] Peverati,C. Translation Education in a Modern-language Faculty Reception and Suitability of Profession-based Workshop Grounded in Social Constructivist Theory[D]. Catholic University of Brescia.2007.

[4] Varney, J. From hermeneutics to the translation classroom: A social constructivist approach to effective learning $[\mathrm{J}]$. The International Journal for Translation and Interpreting Research 2009(1) :12-30.

[5] Du Xiangpei. Research on the evolution of educational thought and practice in women's universities in China [M]. Beijing: Central University for Nationalities Press. 2011. 